\title{
Was there a change in perception of medical students of what good doctors are and what it would take to train them across a 14-year span? A mixed methods panel
}

\author{
Houve uma mudança na percepcao de \\ estudantes de medicina do que significa ser um \\ bom médico e de como educá-lo em um período \\ de 14 anos? Um método de estudo misto
}

Liliana Wahba ${ }^{I}$

Francisco Lotufo ${ }^{I I}$

\section{KEY-WORDS \\ - Medical education; \\ - Medical students; \\ - Physician's role, \\ - Medical ethics.}

\begin{abstract}
Background: Although overall cultural differences exist between individuals from the $X$ and $Y$ generations, to our knowledge few previous studies have contrasted their expectations regarding what good doctors are, and their perceptions on the training processes they undergo. Aims: To conduct a study exploring perceptions on what good doctors are and their perceptions about training among a sample of Brazilian medical students at the University of Sao Paulo Medical School, comparing groups from 2000 and 2014. We aimed to provide information that can be used to guide curriculum development in medical schools. Methods: We conducted a mixed methods study of a series of open questions asked to medical students from the University of São Paulo, Brazil, in 2000 and 2014. This qualitative analysis focused on uncovering emerging themes related to students' perceptions regarding what good doctors are and how they see their training process. A subsequent quantitative analysis through Natural Language Processing was undertaken. Results: Gender distribution was balanced between the 2000 and 2014 groups, with most students being in the early 20s. Our main emerging concepts involved four themes: Skills and qualities of a good doctor, positive and negative aspects of the curriculum, as well as expectations related to students' future career. From a qualitative perspective, the 2014 group focused their criticisms on the School of Medicine itself and the study overload, while the 2000 group focused its criticism on the faculty and competition. Therefore, the 2014 group experienced more criticism and less idealization in relation to the School and the medical training process. There were no statistically significant differences between the 2000 and 2014 groups, as well as across genders. Discussion: Students in this sample have demonstrated little change over time in relation to their characterization of what good doctors are and how they should be trained. Their preferences for a practical, patient-centered education should guide future curriculum development in medical schools.
\end{abstract}

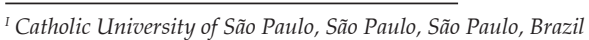

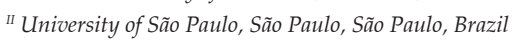




\section{PALAVRAS CHAVE}

- Educação Médica;

- Estudantes de Medicina,

- Papel do Médico,

- Ética Médica.

\section{RESUMO}

Fundamentação: Existem importantes diferenças culturais entre os indivíduos das gerações $x$ e $Y$. Entretanto, poucos estudos compararam as percepções dessas duas gerações em relação ao que são bons médicos, e também quanto ao seu processo de treinamento. Objetivo: Conduzir um estudo explorando as percepções sobre o que significa ser um bom médico e sobre o processo de formação em uma amostra de estudantes de medicina da Faculdade de Medicina da Universidade de São Paulo, comparando grupos de 2000 e de 2014. Buscamos fornecer informações que possam guiar o desenvolvimento curricular em escolas médicas. Método: Foi conduzido um estudo misto por meio de questões abertas dirigidas a estudantes de Medicina da Universidade de São Paulo em 2000 e 2014. A análise qualitativa focou em temas emergentes ligados à percepção dos estudantes sobre o que seriam bons médicos e sobre o treinamento que os estudantes devem receber. Em seguida, foi realizada uma análise quantitativa por meio de Processamento de Linguagem Natural. Resultados: A frequência de homens e mulheres estava balanceada entre os grupos de 2000 e 2014. A maior parte dos estudantes tinha ao redor de 20 anos de idade. Os principais conceitos emergentes encontrados envolveram os quatro temas seguintes: Habilidades $e$ qualidades de um bom médico, aspectos positivos e aspectos negativos do currículo, expectativas relacionadas à carreira futura. Do ponto de vista qualitativo, o grupo de 2014 focou suas críticas na escola médica em si e na excessiva carga de estudo, enquanto o grupo de 2000 criticou os professores e a competitividade. Outrossim, o grupo de 2014 denotou maior criticismo e menor idealização em relação à Escola e ao processo de treinamento. Não houve diferenças estatísticas significativas entre os grupos de 2000 e de 2014, nem entre gêneros. Conclusão: Os estudantes de medicina da FMUSP desta amostra demonstraram pouca mudança no decorrer dos anos em relação ao que consideram ser bons médicos e como eles devem ser treinados. As preferências dos estudantes por uma educação prática e centrada nos pacientes deveriam guiar ofuturo desenvolvimento curricular nas escolas médicas.

Recebido em: 7/10/17

Aceito em: 11/10/17

\section{INTRODUCTION}

The practice of medicine requires the mastering of skills, including but not limited to technical expertise and psychosocial competencies that will support ethical decisions and influence the quality of the doctor-patient relationship ${ }^{1,2}$. As many of these skills are difficult to define and measure, there is an intense discussion on what exactly a good doctor is ${ }^{3,4}$. This concept, complex and multifaceted, means "different things to different people at different times. ${ }^{\prime 5}$ Despite the importance of understanding medical students' expectations to guide the design of educational environments in medical schools, few studies have explored their perspective on this issue ${ }^{6}$. Furthermore, major societal changes in the last decades have not only reshaped the role of medicine, but have also led to a new generation of medical students with a different worldview in several aspects ${ }^{7}$. Although these factors affect the learning experiences and future working lives of physicians-in-training, they have been largely disregarded during the design of medical curricula in Latin America.

Until recent years, most medical students had been born between the 70 s and 80 s, being part of the so-called X Genera- tion (X Gen). Considered transitional, this group has bridged the progression from the analogue to the digital era. As a result, the X Gen does not have an innate understanding of the Internet and other novel technologies ${ }^{8}$. In the professional setting, members of the $X$ Gen were the first to aspire for a balance between hard work and quality of life, while also valuing freedom and flexibility. This trend is in opposition to the workaholic mentality of previous generations ${ }^{9}$. Regarding the current medical students, most of them belong to the $\mathrm{Y}$ generation, the last to achieve age for superior education. These young adults were born between the 80s and the early 90s, being the first to have Internet access from an early age. As a result, they demonstrate a greater degree of engagement with technology, including multitasking habits and widespread exposure to multimedia material ${ }^{10}$. They were also the first true members of a global community, being able to communicate with people around the world. Thus, they have created a more open-minded attitude towards social differences, along with being constantly connected to social networks ${ }^{11}$. These young adults have unique characteristics that affect their attitudes both in school and work environments, such as the preference 
for collaborative learning and interactivity, and the need for constant feedback ${ }^{12}$.

A number of authors have described the need for the medical curriculum to be aligned with cultural changes and expectations from different generations and groups of students ${ }^{13,14}$. This approach can lead to the development of customized, more effective, and pleasant learning environments, as well as to the management of unrealistic ideas. It is however unclear how medical students' expectations have changed across the $X$ and $Y$ generations. Specifically, to our knowledge, there have been nearly no previous studies comparing the views of the $\mathrm{Y}$ and $\mathrm{X}$ generations of medical students regarding what good doctors are, and how to train them in Latin America.

Given this gap in the literature, the objective of this study is to conduct a mixed methods analysis of a series of open questions asked to medical students in 2000 and 2014. Our goal was to evaluate possible changes in relation to their opinions regarding what constitutes a "good doctor," and what might be the requirements to educate such a doctor according to their perceptions, also considering what they might positive and negative in relation to the corresponding school of medicine.

\section{METHODS}

\section{Study design}

This is a mixed-methods research exploring the perception of medical students regarding what good physicians might be, as well as how they see their training process. The survey was conducted twice with a 14-year interval. Our choice for using mixed-methods was aimed at maximizing the interpretation of findings. This study is described in accordance with the Guidelines for Conducting and Reporting Mixed Research in the Field of Counseling and Beyond ${ }^{15}$.

\section{Ethics}

Our study was approved by the Institutional Review Board of the University of São Paulo, informed consent being offered to all potential participants, and subsequently signed prior to any study protocol being implemented.

\section{Setting}

Data were gathered from medical students from the third year (from a six-year program) at the University of São Paulo's School of Medicine, enrolled in the course "Medical Psychology." Data collection occurred in university classrooms in early January of 2000 and 2014. Each questionnaire took approximately twenty to thirty minutes to be responded.

\section{Participants}

All students enrolled in the Medical Psychology course were asked to participate, being informed about the existence of the project during the opening lecture. Potential participants included a total of 169 medical students in $2000(n=98)$ and 162 in $2014(n=64)$. No exclusion criteria were applied. No sociodemographic characteristics were collected from subjects to protect their confidentiality.

\section{Open questions}

The following open questions were posed to each medical student: (1) What are, in your opinion, the qualities of a good doctor? (Quais as qualidades de um bom médico, segundo sua opinião?) (2) Which aspects of your degree program provide a positive contribution to the development of the aforementioned qualities? (Quais aspectos de sua formação médica contribuem positivamente para o desenvolvimento dessas qualidades?) (3) Which aspects of your degree program provide a negative contribution to the development of the aforementioned qualities? (Quais aspectos de sua formação médica contribuem negativamente para o desenvolvimento dessas qualidades?) (4) What are your expectations in relation to your own performance as a physician? (Quais são as expectativas que você tem quanto ao seu desempenho como médico (a)?).

\section{STATISTICAL ANALYSIS}

\section{Qualitative Methods}

We started our study by evaluating students' responses to explore their views and beliefs regarding what a good physician is and the requirements for training this professional. Qualitative data was analyzed based on an inductive approach, specifically through a thematic content analysis. We explored all questionnaires to identify themes and subcategories which were extracted from the responses through coding ${ }^{16}$. From these codes, specific words connected to the main theme were listed, constituting a bridge toward the quantitative analysis.

\section{Quantitative methods}

Our linguistic analysis started with a visual exploration of each variable, a variable being characterized by the presence or absence of each word representing the theme. Independent sub-variables representing a variable were then aggregated using a Boolean, OR combined into a theme-related variable. We then evaluated individual variable frequency, percentage and near-zero variance, missing values and patterns across all variables. In addition, a MINE algorithm was run to guide the bivariate plot inspection. Bivariate comparisons are presented 
in tables without adjustment for multiple testing, and whenever originally statistically-significant, results are adjusted while reporting them in the corresponding text.

Quantitative discourse analysis of response transcripts was conducted to provide frequency counts of statistical stems and their contexts (surrounding terms). Our choice of statistical rather than rule-based stemming, was driven by the relative paucity of adequate computational stemmers for Brazilian Portuguese, in spite of statistical stemming allowing for a more efficient and targeted analysis. All analyses were conducted using the $\mathrm{R}$ statistical language, specifically the qdap package, as well the NLTK (Natural Language Toolkit) within the Python programming language. Cloud plots were used to display word frequencies.

\section{RESULTS}

\section{Emerging themes}

Our analyses identified the following four emerging themes: (1) Qualities of a good doctor, including knowledge, reasoning, scientific aspects, competence, pragmatism, humanism, altruism, love, comprehension, communication, kindness, respect, empathy, sensibility, action, motivation, work, challenges, cases, patients, suffering, ethics, emotions, self-knowledge, creativity, self-confidence, undesirable attributes and cultural attributes; (2) Positive training aspects comprising curriculum, formation, educational methods, school, theoretical, practical, model teacher, model doctor, exemplary, good professional performance, aspirations (medical, surgical and clinical), knowledge, support, humanistic aspects, doctorpatient humanism, cognition/action, subjective-emotional, friendliness, community interactions and university group interactions; (3) Negative aspects of training included arrogance, competition, selfishness, emotional insensibility, course material , workload, professors, quality of life (negative living conditions, negative feelings), role models (doctors, and how medicine is practiced, bad relationships with patients); (4) Expectations and future projects including satisfying investment and gratification (learning, trust, positive emotions, exercising your own profession, treatment and patient care, caring and concern, socialization), insecurity and conflicting emotions.

Below we discuss each of these themes in detail.

\section{Qualities of a good doctor}

There were common themes involving the need for a balance between technical aspects and a good relationship with patients in both groups, recognizing them as individuals with specific needs. There was also an emphasis on ethics, empathy, character building, and dedication to the profession. Less emphasis was placed on professional camaraderie and other social aspects related to medical colleagues.

Quotes related to this theme in 2000 included: “...A good technical base is essential. However, other basic requisites include the ability to not only treat the patients' diseases, but also to know their limitations, and to have compassion," "(...) great theoretical knowledge, great practical knowledge, responsibility, maturity, good group relationships (...)." Then in 2014: "A good physician has good theoretical knowledge, emotional intelligence, and a real concern for patients, having concern with other people and not just with their own egos," "(...) sensibility, ability to see situations as a whole, concern with patients, willing to make a difference, optimism, hope, self-knowledge, dedication, intelligence, good reasoning, and scientific knowledge."

\section{Positive contributors to the development of the aforementioned qualities}

Both groups mentioned the following positive contributors for the development of a good physician: role models provided by professors, family education, personal dedication, and contact with patients. Their discourse also emphasized scientific knowledge, and the companion humanistic component. The 2000 group emphasized the School of Medicine itself, and learning through knowledge. The 2014 group however emphasized on practical classes and learning through enhancing development skills. This group also mentioned the importance of Academic Leagues, activities in which students shadow medical residents and attending physicians during direct patient care as well as sport activities.

Examples of quotes from both groups included: "The University, the classes, etc., help you to be competent. In other words, they help you to learn medicine. What contributes to other qualities such as being patient, attentive, and friendly, are the examples of physicians in practice. For example, one of my professors was the perfect physician in my opinion, he is my role model," "Positive contributors include discussions, experience exchange, and role models from professors."

\section{Negative contributors to the development of the aforementioned qualities}

Negative contributors mentioned by the students included: excessive emphasis on technical rather than humanistic aspects, scarce stimulus to critical thinking, arrogant professors, competition, physical and emotional overload, and low levels of concern for the needs of both patients and students. While the 2014 group focused their criticisms on the School of Medicine itself and the overload caused by the extended number 
of hours dedicated to the course, the 2000 group focused on professors and competition between colleagues, as well as the resulting negative feelings. Examples of quotes in this theme included: "Teachers who do not respect the patients, teachers who act as if they were superior" (2000 and 2014), "In the university there is an atmosphere of competition, arrogance and exaggerated individualism, impairing and leaving in the background qualities such as good manners, humility and gentleness" (2000)," "The immense workload is a negative factor, wearing out students physically and mentally" (2014).

\section{Future expectations}

Regarding future expectations, most students mentioned the aspiration of having professional competence in the care of patients, also describing uncertainty in relation to recognition and income prospects. Particularly among the 2000 group, there was a focus on what we interpreted as a "satisfying investment," including the following components: learning, trust, positive feelings related to the profession, and socialization. The following quotes exemplify this feeling: "A good physician should be respected, while being watchful of the possibility of pride subsuming their other positive traits," and "(It is important to) be a representative of physicians who graduated from this institution, portraying the image of someone who is absolutely trustworthy, well-learned, but also driven by a mission and thus deserving of respect."

\section{Student responses stratified by year}

Most of our students were males, although in 2014 the male to female ratios were the same. Students were averagely 21.2 years old, with the average age increasing by 1.2 years between 2000 and 2014 (20.7 and 21.9, p = 0.276 after multiple adjustments). The two groups did not present a statistically significant difference in relation to the number of words in their responses: $83 \pm 26$ for the 2000 group and $70 \pm 29$ for the 2014 group. Also, an important emphasis was placed by students of both groups on concepts such as good physicians having knowledge $(69.8 \%)$, being focused on patients $(63 \%)$, and also being empathetic (66\%). In relation to its curriculum, both groups believed the 'making' of a good medical doctor should be practical (58\%), and focused on the doctor-patient relationship (57.4\%). Trust was considered a very important positive characteristic $(84.6 \%)$, as well as a focus on treatments $(64.2 \%)$, and caring and concern (82.1\%). After multiple testing adjustments, no significant differences were found between both groups, although unadjusted findings pointed toward differences in relation to a more important role of reasoning among the 2014 group (18.4\% vs $39.1 \%$ ), an increase in problems with the curriculum $(25.5 \%$ vs $48.4 \%)$, a greater perception of excessive workload (3.1\% vs $25 \%$ ), overall negative conditions (6.1\% vs. $26.6 \%)$, as well as an increment in negative feelings toward the curriculum ( $25.5 \%$ vs. $42.2 \%$ ). Although none of these concepts remained statistically-significant after adjustment (all p > 0.05), it was observed through the qualitative analysis that all of them pointed toward an increased degree of dissatisfaction with the curriculum and school conditions, possibly emphasizing that the educational methods might not have kept pace with students' expectations (Table 1). 


\begin{tabular}{|c|c|c|c|c|}
\hline & $\begin{array}{r}\text { TABL } \\
\text { ly sample str }\end{array}$ & d by year & & \\
\hline Concept & Total (162) & Year 2000 (98) & Year 2014 (64) & p \\
\hline Female & $68(42 \%)$ & $36(36.7 \%)$ & $32(50 \%)$ & 0.131 \\
\hline Age & $21.2( \pm 2.42)$ & $20.71( \pm 1.47)$ & $21.95( \pm 3.28)$ & 0.006 \\
\hline QUALITIES OF A GOOD DOC & & & & \\
\hline 1) Relationship characteristics & $151(93.2 \%)$ & $89(90.8 \%)$ & $62(96.9 \%)$ & 0.238 \\
\hline - Empathy & $61(37.7 \%)$ & $35(35.7 \%)$ & $26(40.6 \%)$ & 0.642 \\
\hline - Respect & $60(37 \%)$ & $35(35.7 \%)$ & $25(39.1 \%)$ & 0.791 \\
\hline - Comprehension & $59(36.4 \%)$ & $34(34.7 \%)$ & $25(39.1 \%)$ & 0.691 \\
\hline - Kindness & $50(30.9 \%)$ & $29(29.6 \%)$ & $21(32.8 \%)$ & 0.795 \\
\hline - Sensibility & $30(18.5 \%)$ & $17(17.3 \%)$ & $13(20.3 \%)$ & 0.789 \\
\hline - Humanism & $30(18.5 \%)$ & $22(22.4 \%)$ & $8(12.5 \%)$ & 0.166 \\
\hline - Communication & $18(11.1 \%)$ & $7(7.1 \%)$ & $11(17.2 \%)$ & 0.083 \\
\hline - Love & $10(6.2 \%)$ & $9(9.2 \%)$ & $1(1.6 \%)$ & 0.102 \\
\hline - Altruism & $7(4.3 \%)$ & $2(2 \%)$ & $5(7.8 \%)$ & 0.17 \\
\hline 2) Operational-cognitive & $147(90.7 \%)$ & $92(93.9 \%)$ & $55(85.9 \%)$ & 0.154 \\
\hline - Knowledge & $113(69.8 \%)$ & $72(73.5 \%)$ & $41(64.1 \%)$ & 0.272 \\
\hline - Pragmatism & $56(34.6 \%)$ & $35(35.7 \%)$ & $21(32.8 \%)$ & 0.833 \\
\hline - Competence & $47(29 \%)$ & $33(33.7 \%)$ & $14(21.9 \%)$ & 0.15 \\
\hline - Reasoning & $43(26.5 \%)$ & $18(18.4 \%)$ & $25(39.1 \%)$ & 0.006 \\
\hline - Scientific aspects & $11(6.8 \%)$ & $7(7.1 \%)$ & $4(6.2 \%)$ & 1 \\
\hline 3) Personal characteristics & $135(83.3 \%)$ & $85(86.7 \%)$ & $50(78.1 \%)$ & 0.222 \\
\hline - Emotional & $107(66 \%)$ & $65(66.3 \%)$ & $42(65.6 \%)$ & 1 \\
\hline - Ethics & $52(32.1 \%)$ & $34(34.7 \%)$ & $18(28.1 \%)$ & 0.482 \\
\hline - Self knowledge & $21(13 \%)$ & $17(17.3 \%)$ & $4(6.2 \%)$ & 0.069 \\
\hline - Self confidence & $10(6.2 \%)$ & $8(8.2 \%)$ & $2(3.1 \%)$ & 0.333 \\
\hline - Creativity & $8(4.9 \%)$ & $4(4.1 \%)$ & $4(6.2 \%)$ & 0.801 \\
\hline - Undesirable characteristics & $7(4.3 \%)$ & $5(5.1 \%)$ & $2(3.1 \%)$ & 0.834 \\
\hline 4) Patient and clinic & $114(70.4 \%)$ & $70(71.4 \%)$ & $44(68.8 \%)$ & 0.85 \\
\hline - Patient & $102(63 \%)$ & $64(65.3 \%)$ & $38(59.4 \%)$ & 0.55 \\
\hline - Suffering & $62(38.3 \%)$ & $40(40.8 \%)$ & $22(34.4 \%)$ & 0.51 \\
\hline - Case & $39(24.1 \%)$ & $23(23.5 \%)$ & $16(25 \%)$ & 0.972 \\
\hline 5) Work investment & $71(43.8 \%)$ & $43(43.9 \%)$ & $28(43.8 \%)$ & 1 \\
\hline - Action & $34(21 \%)$ & $18(18.4 \%)$ & $16(25 \%)$ & 0.414 \\
\hline - Motivation & $29(17.9 \%)$ & $19(19.4 \%)$ & $10(15.6 \%)$ & 0.688 \\
\hline - Work & $19(11.7 \%)$ & $12(12.2 \%)$ & $7(10.9 \%)$ & 0.998 \\
\hline - Difficulties & $12(7.4 \%)$ & $6(6.1 \%)$ & $6(9.4 \%)$ & 0.641 \\
\hline 6) Social characteristics & $25(15.4 \%)$ & $16(16.3 \%)$ & $9(14.1 \%)$ & 0.867 \\
\hline - Cultural & $25(15.4 \%)$ & $16(16.3 \%)$ & $9(14.1 \%)$ & 0.867 \\
\hline POSITIVE ASPECTS OF TRAIN & & & & \\
\hline 1) School education & $108(66.7 \%)$ & $67(68.4 \%)$ & $41(64.1 \%)$ & 0.691 \\
\hline - Curriculum & $77(47.5 \%)$ & $45(45.9 \%)$ & $32(50 \%)$ & 0.728 \\
\hline - Formation & $34(21 \%)$ & $23(23.5 \%)$ & $11(17.2 \%)$ & 0.446 \\
\hline - School & $23(14.2 \%)$ & $18(18.4 \%)$ & $5(7.8 \%)$ & 0.099 \\
\hline - Educational methods & $11(6.8 \%)$ & $7(7.1 \%)$ & $4(6.2 \%)$ & 1 \\
\hline 2) Humanism & $106(65.4 \%)$ & $61(62.2 \%)$ & $45(70.3 \%)$ & 0.375 \\
\hline - Humanistic & $43(26.5 \%)$ & $28(28.6 \%)$ & $15(23.4 \%)$ & 0.588 \\
\hline 3) Teaching & $96(59.3 \%)$ & $55(56.1 \%)$ & $41(64.1 \%)$ & 0.4 \\
\hline - Practical & $94(58 \%)$ & $54(55.1 \%)$ & $40(62.5 \%)$ & 0.441 \\
\hline - Theoretical & $6(3.7 \%)$ & $3(3.1 \%)$ & $3(4.7 \%)$ & 0.912 \\
\hline
\end{tabular}




\begin{tabular}{|c|c|c|c|c|}
\hline 4) Qualities & $72(44.4 \%)$ & $46(46.9 \%)$ & $26(40.6 \%)$ & 0.529 \\
\hline - Doctor-patient relationship & $93(57.4 \%)$ & $54(55.1 \%)$ & $39(60.9 \%)$ & 0.567 \\
\hline - Cognition-action & $51(31.5 \%)$ & $31(31.6 \%)$ & $20(31.2 \%)$ & 1 \\
\hline - Subjective-emotional & $43(26.5 \%)$ & $29(29.6 \%)$ & $14(21.9 \%)$ & 0.365 \\
\hline 5) Learning & $70(43.2 \%)$ & $49(50 \%)$ & $21(32.8 \%)$ & 0.046 \\
\hline - Knowledge & $53(32.7 \%)$ & $40(40.8 \%)$ & $13(20.3 \%)$ & 0.011 \\
\hline - Enhancement & $28(17.3 \%)$ & $15(15.3 \%)$ & $13(20.3 \%)$ & 0.541 \\
\hline 6) Group interaction & $65(40.1 \%)$ & $39(39.8 \%)$ & $26(40.6 \%)$ & 1 \\
\hline - Community & $45(27.8 \%)$ & $26(26.5 \%)$ & $19(29.7 \%)$ & 0.796 \\
\hline - Friends & $19(11.7 \%)$ & $14(14.3 \%)$ & $5(7.8 \%)$ & 0.316 \\
\hline - Academic leagues & $8(4.9 \%)$ & $3(3.1 \%)$ & $5(7.8 \%)$ & 0.32 \\
\hline 7) Professional aspirations & $57(35.2 \%)$ & $32(34 \%)$ & $25(36.8 \%)$ & 0.848 \\
\hline - Medicine & $43(26.5 \%)$ & $29(29.6 \%)$ & $14(21.9 \%)$ & 0.365 \\
\hline - Good performance & $22(13.6 \%)$ & $18(18.4 \%)$ & $4(6.2 \%)$ & 0.049 \\
\hline - Clinic & $6(3.7 \%)$ & $2(2 \%)$ & $4(6.2 \%)$ & 0.336 \\
\hline - Surgery & $2(1.2 \%)$ & $0(0 \%)$ & $2(3.1 \%)$ & 0.302 \\
\hline 8) Medical models & $50(30.9 \%)$ & $28(28.6 \%)$ & $22(34.4 \%)$ & 0.543 \\
\hline - Doctor & $35(21.6 \%)$ & $23(23.5 \%)$ & $12(18.8 \%)$ & 0.604 \\
\hline - Teacher & $25(15.4 \%)$ & $20(20.4 \%)$ & $5(7.8 \%)$ & 0.052 \\
\hline - Example & $14(8.6 \%)$ & $9(9.2 \%)$ & $5(7.8 \%)$ & 0.986 \\
\hline \multicolumn{5}{|l|}{ NEGATIVE ASPECTS OF TRAINING } \\
\hline 1) School training & $74(45.7 \%)$ & $34(34.7 \%)$ & $40(62.5 \%)$ & $<0.001$ \\
\hline - Course & $56(34.6 \%)$ & $25(25.5 \%)$ & $31(48.4 \%)$ & 0.005 \\
\hline - Workload & $19(11.7 \%)$ & $3(3.1 \%)$ & $16(25 \%)$ & $<0.001$ \\
\hline - Teachers & $17(10.5 \%)$ & $13(13.3 \%)$ & $4(6.2 \%)$ & 0.245 \\
\hline 2) Quality of life & $60(37 \%)$ & $27(27.6 \%)$ & $33(51.6 \%)$ & 0.003 \\
\hline - Negative feelings & $52(32.1 \%)$ & $25(25.5 \%)$ & $27(42.2 \%)$ & 0.04 \\
\hline - Negative conditions & $23(14.2 \%)$ & $6(6.1 \%)$ & $17(26.6 \%)$ & $<0.001$ \\
\hline 3) Medical models & $50(30.9 \%)$ & $28(28.6 \%)$ & $22(34.4 \%)$ & 0.543 \\
\hline - Professional practice & $33(20.4 \%)$ & $18(18.4 \%)$ & $15(23.4 \%)$ & 0.559 \\
\hline - Doctor-patient relationship & $31(19.1 \%)$ & $15(15.3 \%)$ & $16(25 \%)$ & 0.184 \\
\hline - Doctor & $21(13 \%)$ & $12(12.2 \%)$ & $9(14.1 \%)$ & 0.922 \\
\hline 4) Negative characteristics in students and doctors & $31(19.1 \%)$ & $23(23.5 \%)$ & $8(12.5 \%)$ & 0.126 \\
\hline - Lack of sensibility & $13(8 \%)$ & $9(9.2 \%)$ & $4(6.2 \%)$ & 0.707 \\
\hline - Arrogance & $11(6.8 \%)$ & $8(8.2 \%)$ & $3(4.7 \%)$ & 0.589 \\
\hline - Competition & $9(5.6 \%)$ & $9(9.2 \%)$ & $0(0 \%)$ & 0.032 \\
\hline - Emotional & $4(2.5 \%)$ & $3(3.1 \%)$ & $1(1.6 \%)$ & 0.934 \\
\hline - Selfishness & $4(2.5 \%)$ & $4(4.1 \%)$ & $0(0 \%)$ & 0.263 \\
\hline \multicolumn{5}{|l|}{ FUTURE EXPECTATIONS } \\
\hline 1) Satisfying investment & $160(98.8 \%)$ & $98(100 \%)$ & $62(96.9 \%)$ & 0.302 \\
\hline - Trust & $137(84.6 \%)$ & $80(81.6 \%)$ & $57(89.1 \%)$ & 0.29 \\
\hline - Caring and concern & $133(82.1 \%)$ & $81(82.7 \%)$ & $52(81.2 \%)$ & 0.986 \\
\hline - Practice & $131(80.9 \%)$ & $80(81.6 \%)$ & $51(79.7 \%)$ & 0.918 \\
\hline - Treatment of patients & $104(64.2 \%)$ & $61(62.2 \%)$ & $43(67.2 \%)$ & 0.636 \\
\hline - Learning and training & $75(46.3 \%)$ & $49(50 \%)$ & $26(40.6 \%)$ & 0.313 \\
\hline - Positive feelings about profession & $36(22.2 \%)$ & $19(19.4 \%)$ & $17(26.6 \%)$ & 0.379 \\
\hline - Socialization & $26(16 \%)$ & $17(17.3 \%)$ & $9(14.1 \%)$ & 0.735 \\
\hline 2) Insecurity and conflictive emotions & $45(27.8 \%)$ & $27(27.6 \%)$ & $18(28.1 \%)$ & 1 \\
\hline
\end{tabular}

Significant p-values were marked in bold (before multiple testing adjustments).

\section{Student responses stratified by gender}

When comparing male and female students, the only statistically-significant difference between them was that the latter were younger, although this difference lost its significance after adjustment for multiple testing (Table A 1 in our Appendix). 


\section{TABle A1}

\section{Study sample stratified by gender}

\section{Concept}

Age

\section{QUALITIES OF A GOOD DOCTOR}

1) Operational-cognitive

- Knowledge

- Pragmatism

- Reasoning

- Competence

- Scientific Aspects

2) Relationship characteristics

- Empathy

- Respect

- Comprehension

- Kindness

- Humanism

- Sensibility

- Communication

- Love

- Altruism

3) Personal characteristics

- Action

- Motivation

- Work

- Difficulties

4) Patient and clinic

- Patient

- Suffering

- Case

5) Work investment

- Action

- Motivation

- Work

- Difficulties

6) Social characteristics

- Cultural

POSITIVE ASPECTS OF TRAINING

1) School education

- Curriculum

- Formation

- School

- Educational methods

2) Humanism

- Doctor-patient relationship

- Humanistic

3) Teaching

- Practical

- Theoretical
Male students (94)

$21.52( \pm 2.83)$

$87(92.6 \%)$

$66(70.2 \%)$

$33(35.1 \%)$

$26(27.7 \%)$

$24(25.5 \%)$

$6(6.4 \%)$

$86(91.5 \%)$

$36(38.3 \%)$

34 (36.2 \%)

$31(33 \%)$

$28(29.8 \%)$

$14(14.9 \%)$

$13(13.8 \%)$

$12(12.8 \%)$

$7(7.4 \%)$

$4(4.3 \%)$

$73(77.7 \%)$

$18(19.1 \%)$

15 (16\%)

$12(12.8 \%)$

$7(7.4 \%)$

$61(64.9 \%)$

$52(55.3 \%)$

$35(37.2 \%)$

$20(21.3 \%)$

$38(40.4 \%)$

$18(19.1 \%)$

$15(16 \%)$

$12(12.8 \%)$

$7(7.4 \%)$

$14(14.9 \%)$

$14(14.9 \%)$

$63(67 \%)$

$44(46.8 \%)$

$20(21.3 \%)$

$11(11.7 \%)$

$8(8.5 \%)$

$57(60.6 \%)$

$48(51.1 \%)$

$30(31.9 \%)$

$53(56.4 \%)$

$51(54.3 \%)$

$5(5.3 \%)$

\section{Female students (68)}

$20.76( \pm 1.64)$

$60(88.2 \%)$

$47(69.1 \%)$

$23(33.8 \%)$

$17(25 \%)$

$23(33.8 \%)$

$5(7.4 \%)$

$65(95.6 \%)$

$25(36.8 \%)$

$26(38.2 \%)$

$28(41.2 \%)$

$22(32.4 \%)$

$16(23.5 \%)$

$17(25 \%)$

$6(8.8 \%)$

$3(4.4 \%)$

$3(4.4 \%)$

$62(91.2 \%)$

$16(23.5 \%)$

$14(20.6 \%)$

$7(10.3 \%)$

$5(7.4 \%)$

$53(77.9 \%)$

$50(73.5 \%)$

$27(39.7 \%)$

$19(27.9 \%)$

$33(48.5 \%)$

$16(23.5 \%)$

$14(20.6 \%)$

$7(10.3 \%)$

$5(7.4 \%)$

$11(16.2 \%)$

$11(16.2 \%)$

$45(66.2 \%)$

$33(48.5 \%)$

$14(20.6 \%)$

$12(17.6 \%)$

$3(4.4 \%)$

$49(72.1 \%)$

$45(66.2 \%)$

13 (19.1\%)

$43(63.2 \%)$

$43(63.2 \%)$

$1(1.5 \%)$ p-value

0.034

0.509

0.998

0.843

0.331

1

0.48

0.972

0.917

0.366

0.86

0.233

0.109

0.593

0.644

1

0.039

0.631

0.582

0.814

1

0.105

0.028

0.876

0.428

0.387

0.631

0.582

0.814

1

0.998

0.998

\section{1}

0.954

1

0.4

0.48

0.18

0.079

0.101

0.475

0.326

0.391 


\begin{tabular}{|c|c|c|c|}
\hline 4) Learning & $49(52.1 \%)$ & $21(30.9 \%)$ & 0.011 \\
\hline - Knowledge & $38(40.4 \%)$ & $15(22.1 \%)$ & 0.022 \\
\hline - Enhancement & $19(20.2 \%)$ & $9(13.2 \%)$ & 0.343 \\
\hline 5) Qualities & $42(44.7 \%)$ & $30(44.1 \%)$ & 1 \\
\hline - Cognition-action & $28(29.8 \%)$ & $23(33.8 \%)$ & 0.708 \\
\hline - Subjective-emotional & $26(27.7 \%)$ & $17(25 \%)$ & 0.843 \\
\hline 6) Group interaction & $35(37.2 \%)$ & $30(44.1 \%)$ & 0.472 \\
\hline - Community & $22(23.4 \%)$ & $23(33.8 \%)$ & 0.199 \\
\hline - Friends & $11(11.7 \%)$ & $8(11.8 \%)$ & 1 \\
\hline - Academic leagues & $4(4.3 \%)$ & $4(5.9 \%)$ & 0.917 \\
\hline 7) Professional aspirations & $32(34 \%)$ & $25(36.8 \%)$ & 0.848 \\
\hline - Medicine & $23(24.5 \%)$ & $20(29.4 \%)$ & 0.601 \\
\hline - Good performance & $12(12.8 \%)$ & $10(14.7 \%)$ & 0.902 \\
\hline - Clinic & $2(2.1 \%)$ & $4(5.9 \%)$ & 0.408 \\
\hline - Surgery & $1(1.1 \%)$ & $1(1.5 \%)$ & 1 \\
\hline 8) Medical models & $28(29.8 \%)$ & $22(32.4 \%)$ & 0.86 \\
\hline - Doctor & $19(20.2 \%)$ & $16(23.5 \%)$ & 0.754 \\
\hline - Teacher & $11(11.7 \%)$ & $14(20.6 \%)$ & 0.185 \\
\hline - Example & $7(7.4 \%)$ & $7(10.3 \%)$ & 0.724 \\
\hline \multicolumn{4}{|l|}{ NEGATIVE ASPECTS OF TRAINING } \\
\hline 1) School training & $41(43.6 \%)$ & $33(48.5 \%)$ & 0.646 \\
\hline - Course & $34(36.2 \%)$ & $22(32.4 \%)$ & 0.736 \\
\hline - Teachers & $11(11.7 \%)$ & $6(8.8 \%)$ & 0.741 \\
\hline - Workload & $9(9.6 \%)$ & $10(14.7 \%)$ & 0.451 \\
\hline 2) Quality of life & $33(35.1 \%)$ & $27(39.7 \%)$ & 0.665 \\
\hline - Negative feelings & $30(31.9 \%)$ & $22(32.4 \%)$ & 1 \\
\hline - Negative conditions & $11(11.7 \%)$ & $12(17.6 \%)$ & 0.4 \\
\hline 3) Medical models & $28(29.8 \%)$ & $22(32.4 \%)$ & 0.86 \\
\hline - Professional practice & $19(20.2 \%)$ & $14(20.6 \%)$ & 1 \\
\hline - Doctor-patient relationship & $16(17 \%)$ & $15(22.1 \%)$ & 0.547 \\
\hline - Doctor & $10(10.6 \%)$ & $11(16.2 \%)$ & 0.424 \\
\hline 4) Negative characteristics in students and doctors & $20(21.3 \%)$ & $11(16.2 \%)$ & 0.541 \\
\hline - Lack of sensibility & $9(9.6 \%)$ & $4(5.9 \%)$ & 0.575 \\
\hline - Arrogance & $6(6.4 \%)$ & $5(7.4 \%)$ & 1 \\
\hline - Competition & $6(6.4 \%)$ & $3(4.4 \%)$ & 0.847 \\
\hline - Emotional & $2(2.1 \%)$ & $2(2.9 \%)$ & 1 \\
\hline - Selfishness & $2(2.1 \%)$ & $2(2.9 \%)$ & 1 \\
\hline \multicolumn{4}{|l|}{ FUTURE EXPECTATIONS } \\
\hline 1) Satisfying investment & $94(100 \%)$ & $66(97.1 \%)$ & 0.341 \\
\hline - Trust & $81(86.2 \%)$ & $56(82.4 \%)$ & 0.657 \\
\hline - Practice & $80(85.1 \%)$ & $51(75 \%)$ & 0.158 \\
\hline - Learning and training & $40(42.6 \%)$ & $35(51.5 \%)$ & 0.335 \\
\hline - Positive feelings about profession & $17(18.1 \%)$ & $19(27.9 \%)$ & 0.194 \\
\hline - Caring and concern & $12(12.8 \%)$ & $14(20.6 \%)$ & 0.262 \\
\hline 2) Insecurity and conflictive emotions & $23(24.5 \%)$ & $22(32.4 \%)$ & 0.353 \\
\hline
\end{tabular}

Significant $\mathrm{p}$-values were marked in bold (before multiple testing adjustment 


\section{Word clouds for each question}

The word clouds for each of the questions largely reflect the previously described concepts, with a strong emphasis on patients (represented by the stem "paci"), knowledge (conhec, sab), and technical aspects (tecn). Positive qualities are emphasized by concepts such as contact with patients (contat paci), classes (aul), knowledge (conhec), practice (prat), and development (desenvolv) (Figure 1).

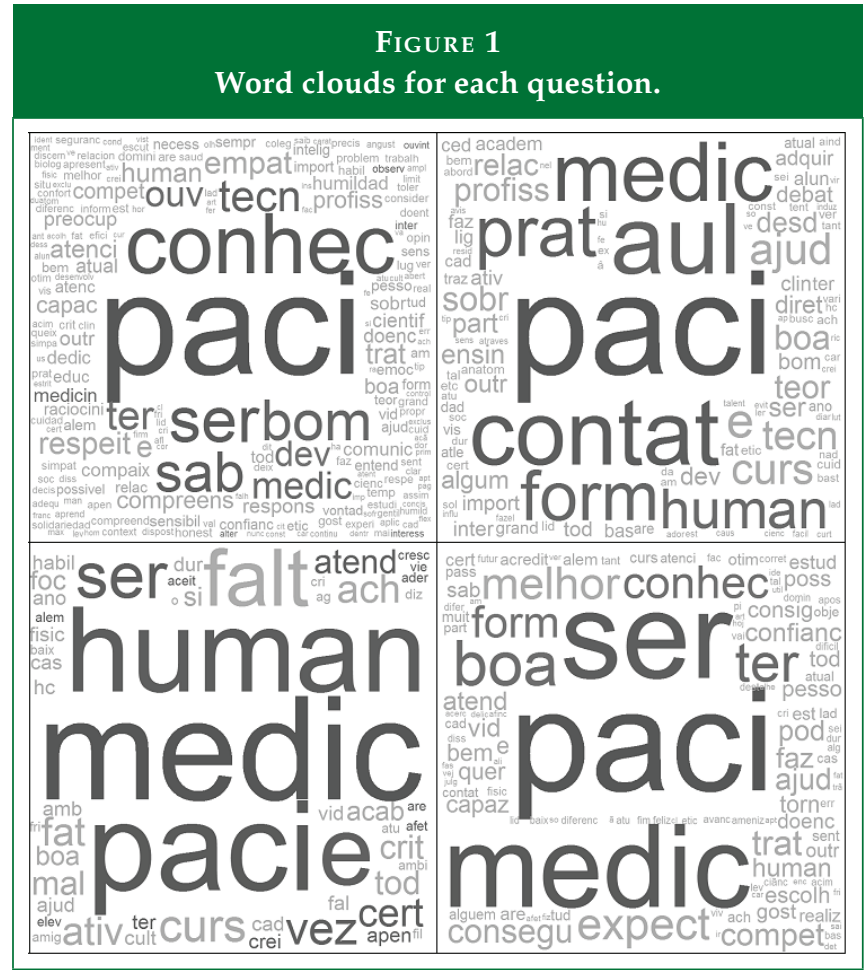

Word clouds, in sequence, for qualities of a good doctor, positive aspects of training, negative aspects of training, future expectations.

Overlap in the content of all word stems for the overall question, comparing 2000 and 2014

When observing the overall overlap in concepts regarding the 2000 and 2014 groups, there was a substantial degree of congruency in their perceptions and expectations, which agreed with our previous analyses (Figure 2). Specifically, the two groups of students presented an overlap of $83.2 \%$ in relation to their concepts, meaning that they represented similar ideas that have therefore not substantially changed in the interval studies in this project.

\section{DISCUSSION}

To our knowledge, this is the first study comparing two generations of medical students in relation to their opinions regarding what constitutes a "good doctor, and how they see
Figure 2

Venn diagram comparing the 2000 and 2014

groups in their perceptions of what good doctors are and their training process.

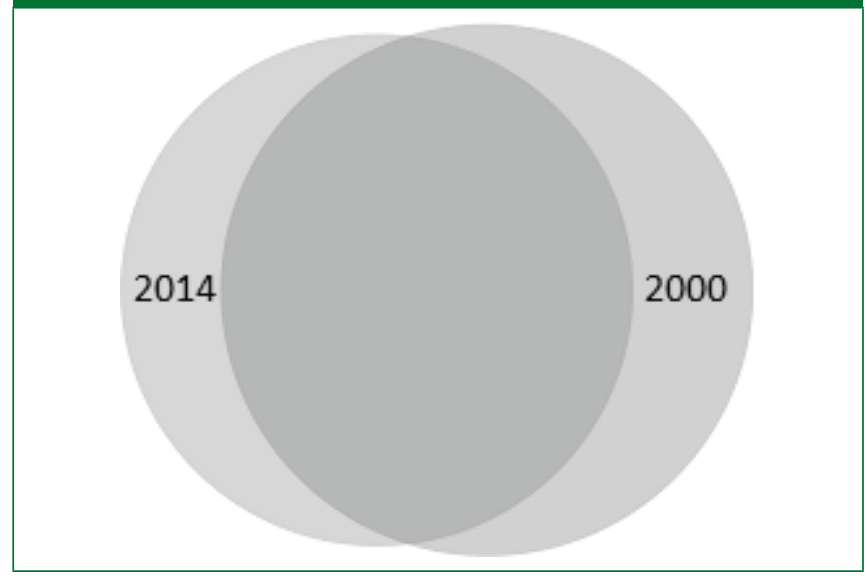

positive and negative aspects of their medical education." Our main concepts constituted the following four themes: Skills and qualities of a good doctor, positive and negative aspects of the curriculum, as well as expectations related to the future career. From a qualitative perspective, the 2014 group focused their criticisms on the School of Medicine itself and the overload caused by the extended number of hours dedicated to the course, whereas the 2000 group focused its criticism on the faculty and competition, although they seemed to be proud of their School. Therefore, the 2014 group experienced more criticism and less idealization in relation to the School and the medical training process. From a quantitative perspective, there were no statistically significant differences between the expectations of medical students over a 14-year span in any of the four thematic groups, including skills and qualities of a good doctor, positive and negative training aspects, and expectations and future projects.

Our results might reflect intersections in the mindset of individuals born in two contiguous generations in relation to basic values of the medical profession. It is worth noting that pointing out the differences between generational groups has for so long been the focus of previous research ${ }^{17,18}$. However, in agreement with our findings, a longitudinal study with a large sample of Australian individuals revealed several points of agreement between the $X$ and $Y$ Gens, such as the concern with balancing professional and personal lives, and the desire for careers that lead to personal fulfillment ${ }^{13}$. Given these findings, there is a need for caution towards the stereotypical notion that these generations are radically shifting in every aspect. Summarily, the major criterion of separation between 
both generations was age. The $\mathrm{X}$ Generation constituted medical students born between the 70s and 80s, whereas the $Y$ Generation constituted those born between the 80 s and the early 90s (age ranges 16-37). Other distinguishing criteria reported in the literature include Y Generation's preference for collaborative learning and interactivity, their greater degree of engagement with technology, value for speed, multitasking habits, widespread exposure to multimedia, and their need for constant feedback, as opposed to the X Generation's preference for efficiency over speed, lack of an innate understanding of the Internet and other novel technologies.

Another possible explanation for our findings is bias among students accepted in medical schools. In most countries, including Brazil, being approved for a medical school is specially challenging, requiring an excellent performance ${ }^{19}$. It has been demonstrated that academic success is not only dependent on acquiring theoretical knowledge, but also on role mastery, defined as a familiarity with what is expected from a student in different educational settings. This ability is associated with an enhanced capacity to recognize and respond to these expectations ${ }^{20}$. It is therefore possible that in having high levels of role mastery, both generations of medical students provided responses based on their tacit knowledge of what is traditionally expected of a good doctor in academic environments. In contrast with our findings, previous studies demonstrated variability in expectations in relation to the gender of medical students, with women tending to seek more balance between work and personal life, expressing increased concern regarding the tensions between career and family ${ }^{21}$. In both genders, these uncertainties tend to decrease over the course of the degree program, perhaps due to an increasing amount of information being gathered over time ${ }^{22}$.

It is believed that students aim for a combination of the characteristics of 'someone who is friendly' and 'someone who listens,' side by side with 'somebody who is intelligent and bright' and 'somebody who is knowledgeable about medical matters. ${ }^{23}$ In agreement with our results, it is agreed that students around the globe expect medicine to be fulfilling both intellectually and personally, the combination of humanistic and technical skills being a major goal ${ }^{24}$. Our results also align with the overarching conclusions from a 2010 systematic review, compiling information on medical professionalism as perceived by medical students, which concluded that professionalism is a multifaceted concept, and its translation to medical curricula a challenge. Furthermore, despite the wide range of methodological approaches, the absence of evidence-based strategies for the teaching and assessment of professionalism in future doctors was demonstrated ${ }^{25}$. In addition to these as- pects, our study also emphasized that students expect the faculty to be closer to them, with a greater emphasis in pragmatic aspects, group work and construction of knowledge $\mathrm{e}^{26}$.

The 2014 group seems to have experienced a decline in idealization in relation to the medical school and training process, identified through our qualitative analysis. This is of concern since a positive attitude toward psycho-social aspects of the medical profession has been associated with a greater degree of communication with patients as well as humanistic traits during the provision of care ${ }^{27}$. Our findings are not without precedent, however, as a decline in idealism during the medical course and internship periods has been previously identified $^{28}$. This decline is particularly evident after the first couple years ${ }^{29,30}$ when students are confronted with the massive amount of technology, patient volume, disease severity, financial constraints, and a lack of role models that would perhaps emphasize their continued focus on humanistic aspects of patient care $^{31}$. Another finding of our qualitative analysis is that 2000 students seemed more proud of their school, also showing higher expectations of gratification. The skepticism and decline in idealization in the 2014 group, although undesirable to some extent, might be compensated by the satisfaction obtained through Academic Leagues and collaborative learning, perhaps displaying a trend that is characteristic of the $\mathrm{Y}$ generation.

The blend of skills expected from a good doctor has important implications for medical schools, as humanistic and technical qualities seem to be integrated in students' minds as being part of a physician's training process. While how exactly this merge can be accomplished is debatable, modern education methodologies suggest that complex educational environments and whole tasks should be explored ${ }^{32}$. It is also important to emphasize that curriculum design should take into account the high degree of stress among students in medical school $^{33}$. A Curriculum reform, aimed at providing students with free time for extracurricular activities such as scientific research, academic extension, and sports ${ }^{34}$, was implemented at the University of São Paulo's School of Medicine in 2015, by reducing their workload by approximately $30 \%$. Factors accounting for this workload as placed in evidence by our study include the long hours, constant exams, need of financial support from parents or others, intense level of responsibilities, mental and physical exhaustion, sleep deprivation, in addition to contact with ill and terminal patients ${ }^{35,36}$. This environment can often lead to the use of alcohol and illicit drugs as an escape mechanism ${ }^{37}$.

Of importance, limitations in our methodology might have impaired the sensitivity of our study to detect significant dif- 
ferences between the generations. First, our sample size was relatively small, and therefore our study may not have had sufficient statistical power for detecting differences between groups. Second, our interview technique may not have effectively mapped students' cognitive schemata regarding their perceptions. Third, students in our sample might not have felt sufficiently motivated or comfortable to share certain aspects of their opinions, perhaps for fear of being judged. Finally, it is also possible that the way the data were organized in our linguistic analysis did not capture subtle aspects of students' reports.

In conclusion, our study did not find statistically-significant differences in the expectations of the $X$ and $Y$ generations of medical students regarding what a good doctor is, and how to train one. However, qualitative findings call attention to the importance of medical schools adapting teaching methods to the learning style of the $\mathrm{Y}$ generation. Our findings also highlight the importance of structuring the medical curriculum according to students' expectations, considering not only differences among generations, but also points of agreement, such as the preference for a practical, patient-centered education focusing on both technical and humanistic aspects of the profession. In the future, longitudinal studies with larger samples of medical students should be performed in order to verify generational and subgroup differences on expectations regarding different aspects of the medical carrier ${ }^{17,38}$.

\section{REFERENCES}

1. Rosenbaum ME, Ferguson KJ, Lobas JG. Teaching medical students and residents skills for delivering bad news: A review of strategies. Acad Med. 2004;79(2):107-17.

2. Deveugele M, Derese A, De Maesschalck S, Willems S, Van Driel M, De Maeseneer J. Teaching communication skills to medical students, a challenge in the curriculum? Patient Educ Couns. 2005;58(3):265-70.

3. Coulter A. Editorials: Patients' views of the good doctor. Student BMJ. 2002;10:400.

4. Hurwitz B, Vass A. What's a good doctor, and how can you make one? BMJ. 2002;325(7366):667-8.

5. Tonks A. What is a good doctor and how do you make one. BMJ. 2002;325:711.

6. Sander P, Stevenson K, King M, Coates D. University students' expectations of teaching. Stud High Educ. 2000;25(3):309-23.

7. Jones R, Higgs R, Angelis C de, Prideaux D. Changing face of medical curricula. Lancet. 2001;357(9257):699-703.

8. Generation X - National Geographic Channel. 2016 [cited 2016 Jun 8]. Available from: http://channel.nationalgeographic.com/generation-x/
9. O’Bannon G. Managing our future: The generation $x$ factor. Public Pers Manage. 2001;30(1):95-110.

10. Sandars J, Morrison C. What is the net generation? The challenge for future medical education. Med Teach. 2007;29(2-3):85-8

11. DiLullo C, McGee P, Kriebel RM. Demystifying the millennial student: A reassessment in measures of character and engagement in professional education. Anat Sci Educ. 2011;4(4):214-26.

12. Eckleberry-Hunt J, Tucciarone J. The challenges and opportunities of teaching "Generation Y". J Grad Med Educ. 2011 Dec;3(4):458-61.

13. McLean M, Johnson P, Sargeant S, Green P. Simulated patients' perspectives of and perceived role in medical students' professional identity development. Simul Healthc. 2015 Apr;10(2):85-91.

14. Wilson I, Cowin LS, Johnson M, Young H. Professional identity in medical students: Pedagogical challenges to medical education. Teach Learn Med. 2013;25(4):369-73.

15. Leech NL, Onwuegbuzie AJ. Guidelines for conducting and reporting mixed research in the field of counseling and beyond. J Couns Dev. 2010;88(1):61.

16. Burnard P, Gill P, Stewart K, Treasure E, Chadwick B. Analysing and presenting qualitative data. Br Dent J. 2008 Apr;204(8):429-32.

17. Moreno-Walton L, Brunett P, Akhtar S, DeBlieux P. Teaching across the generation gap: A consensus from the council of emergency medicine residency directors 2009 academic assembly. Acad Emerg Med. 2009;16(s2):S19-24.

18. Borges NJ, Manuel RS, Elam CL, Jones BJ. Comparing millennial and generation $\mathrm{x}$ medical students at one medical school. Acad Med. 2006;81(6):571-6.

19. G1. Medicina em Ribeirão Preto é o curso mais concorrido da Fuvest 2016. 2015 [cited 2016 Jun 11]. Available from: http://g1.globo.com/educacao/noticia/2015/11/ medicina-em-ribeirao-preto-e-o-curso-mais-concorrido-da-fuvest-2016.html

20. Collier PJ, Morgan DL. "Is that paper really due today?": Differences in first-generation and traditional college students' understandings of faculty expectations. J High Educ. 2008;55(4):425-46.

21. Drinkwater J, Tully MP, Dornan T. The effect of gender on medical students' aspirations: A qualitative study. Med Educ. 2008;42(4):420-6.

22. Maudsley G, Williams L, Taylor D. Medical students' and prospective medical students' uncertainties about career intentions: Cross-sectional and longitudinal studies. Med Teach. 2010;32(3):e143-51. 
23. Leahy M, Cullen W, Bury G. "What makes a good doctor?" a cross sectional survey of public opinion. Ir Med J. 2003;96(2):38-41.

24. Gillies RA, Warren PR, Messias E, Salazar WH, Wagner PJ, Huff TA, et al. Why a medical career and what makes a good doctor? Beliefs of incoming United States medical students. Educ Health. 2009;22(3):331.

25. Passi V, Doug M, Peile JT, Johnson N. Developing medical professionalism in future doctors: A systematic review. Int J Med Educ. 2010;1:19.

26. Souza C da S, Iglesias AG, Pazin-Filho A. Estratégias inovadoras para métodos de ensino tradicionais: Aspectos gerais. Medicina (Ribeirao Preto). 2014;47(3):284-92.

27. Rogers JC, Coutts L. Do students' attitudes during preclinical years predict their humanism as clerkship students? Acad Med. 2000;75(10):S74-7.

28. Morley CP, Roseamelia C, Smith JA, Villarreal AL. Decline of medical student idealism in the first and second year of medical school: A survey of pre-clinical medical students at one institution. Med Educ Online. 2013;18.

29. Campos JADB, Jordani PC, Zucoloto ML, Bonafé FSS, Maroco J. Burnout syndrome among dental students. Rev Bras Epidemiol. 2012;15(1):155-65.

30. Alves JG, Tenório M, Anjos AG dos, Figueroa JN. Qualidade de vida em estudantes de medicina no início e final do curso: Avaliação pelo whoqol-bref. Rev Bras Educ Med. 2010;34(1):91-6.

31. Kay J. Traumatic deidealization and the future of medicine. Jama. 1990;263(4):572-3.

32. Van Merriënboer JJ, Kirschner PA. Ten steps to complex learning: A systematic approach to four-component instructional design. Routledge; 2012.

33. Brock D, Abu-Rish E, Chiu C-R, Hammer D, Wilson S, Vorvick $\mathrm{L}$, et al. Interprofessional education in team communication: Working together to improve patient safety. BMJ Qual Saf. 2013;22(5):414-23.
34. Graduação FMUSP [cited 2016 Aug 5]. Available from: http:/ / www2.fm.usp.br/graduacao/

35. RezendeI CHA de, AbrãoI CB, CoelhoI EP, Silva PassosI LB da. Prevalência de sintomas depressivos entre estudantes de medicina da Universidade Federal de Uberlândia. Rev Bras Educ Med. 2008;32(3):315-23.

36. Rosa EA, Simões JC. O universo psicológico do futuro médico. Rev Med Res. 2013;15(1).

37. Rocha LA, ACFMM L, Martelli DRB, Lima VB, Martelli-Júnior $\mathrm{H}$. Consumo de álcool entre estudantes de faculdades de medicina de Minas Gerais, Brasil. Rev Bras Educ Med. 2011;35(3):369-75.

38. Vanderveen K, Bold RJ. Effect of generational composition on the surgical workforce. Arch Surg. 2008;143(3):224-6.

\section{AUTHOR AFFILIATIONS:}

${ }^{1}$ Post Graduate Program of Clinical Psychology - Catholic University of São Paulo, Rua Ministro Godoy 969, 05015-000, São Paulo-SP, Brazil

${ }^{2}$ Department and Institute of Psychiatry - University of São Paulo, Rua Dr Ovidio Pires de Campos 785, 05403-903, São Paulo-SP, Brasil

\section{CONFLITO DE INTERESSE}

Nao existe conflito de interesses

\section{CORRESPONDING AUTHOR:}

Liliana Liviano Wahba. Post Graduate Program of Clinical Psychology - Catholic University of São Paulo, Rua Ministro Godoy 969, 05015-000, São Paulo-SP, Brazil, 1136735091 lilwah@uol.com.br

\section{AUTHOR CONTRIBUTION:}

All authors contributed with the following tasks for this project: Analysis and interpretation, drafting of the manuscript, reviewing, and the final approval of the material. 